\title{
Early spread of the 2009 infuenza A(H1N1) pandemic in the United Kingdom - use of local syndromic data, May-August 2009
}

S Smith (sue.smith@hpa.org.uk) ${ }^{1}$, G E Smith ${ }^{1}$, B Olowokure ${ }^{2}$, S Ibbotson ${ }^{1}$, D Foord ${ }^{3}$, H Maguire ${ }^{4}$, R Pebody $^{5}$, A Charlett $^{4}$, J Hippisley-Cox ${ }^{6}$, J Elliot ${ }^{1}$

1. Real-time Syndromic Surveillance Team, Health Protection Agency, Birmingham, United Kingdom

2. West Midlands Regional Epidemiology Unit, Health Protection Agency, Birmingham, United Kingdom

3. NHS Direct, Linford Wood East, Milton Keynes, United Kingdom

4. Health Protection Agency London, Regional Epidemiology Unit, London, United Kingdom

5. Health Protection Agency, Centre for Infections, London, United Kingdom

6. Division of Primary Care, School of Community Health Sciences, University of Nottingham, Nottingham, United Kingdom

Citation style for this article:

Smith S, Smith GE, Olowokure B, Ibbotson S, Foord D, Maguire H, Pebody R, Charlett A, Hippisley-Cox J, Elliot AJ. Early spread of the 20og infuenza A(H1N1) pandemic in the United Kingdom - use of local syndromic data, May-August 2009. Euro Surveill. 2011;16(3):pii=19771. Available online: http://www.

eurosurveillance.org/ViewArticle.aspx?Articleld=19771

Following the confirmation of the first two cases of pandemic influenza on 27 April 2009 in the United Kingdom (UK), syndromic surveillance data from the Health Protection Agency (HPA)/QSurveillance and HPA/NHS Direct systems were used to monitor the possible spread of pandemic influenza at local level during the first phase of the outbreak. During the early weeks, syndromic indicators sensitive to influenza activity monitored through the two schemes remained low and the majority of cases were travel-related. The first evidence of community spread was seen in the West Midlands region following a school-based outbreak in central Birmingham. During the first phase several Primary Care Trusts had periods of exceptional influenza activity two to three weeks ahead of the rest of the region. Community transmission in London began slightly later than in the West Midlands but the rates of influenza-like illness recorded by general practitioners (GPs) were ultimately higher. Influenza activity in the West Midlands and London regions peaked a week before the remainder of the UK. Data from the HPA/NHS Direct and HPA/QSurveillance systems were mapped at local level and used alongside laboratory data and local intelligence to assist in the identification of hotspots, to direct limited public health resources and to monitor the progression of the outbreak. This work has demonstrated the utility of local syndromic surveillance data in the detection of increased transmission and in the epidemiological investigation of the pandemic and has prompted future spatio-temporal work.

\section{Introduction}

The first two cases of pandemic influenza in the United Kingdom (UK) were confirmed in Scotland on 27 April 2009 [1]. Initially UK policy was to contain the spread of the virus and during the early stages the main focus of surveillance was on virologically confirmed cases. This containment policy continued until 2 July when the Government announced that due to further spread of the disease the UK was moving to a treatment (mitigation) phase [2]. A key factor in this decision was the presence of sustained community transmission. Data from a range of national surveillance systems, including syndromic surveillance data, were used during the pandemic to assess when the change from sporadic cases to more widespread community transmission occurred.

Syndromic surveillance systems monitor generic symptoms and/or clinically diagnosed disease in order to provide timely information at an earlier stage of illness (compared to laboratory-confirmed diagnosis) [3]. Data are captured electronically, often using information collected for other purposes, to create large datasets that can be analysed rapidly, some systems being able to provide daily data. Some systems are well established, for example the Royal College of General Practitioners Weekly Returns Service has many years of historical data that can be used to monitor longer-term disease trends $[4,5]$. Syndromic surveillance can provide early warning of, for example, seasonal rises in influenza and norovirus infections and can trigger appropriate public health action but can also be used to alert to unexpected events such as an unusual rise in illness that could indicate an outbreak $[6,7]$.

This paper describes the early spread of influenza-like illness (ILI) at Primary Care Trust (PCT) level during the first phase of the 2009 influenza pandemic using data from national syndromic surveillance systems, with a particular focus on West Midlands and London, the areas initially most affected, in order to identify the point when sustained community transmission began.

\section{Methods}

HPA/NHS Direct surveillance system

NHS Direct is a 24 -hour nurse-led telephone helpline that provides health information and advice to the 
general public [8]. To handle the calls, nurses use a computerised clinical decision support system that uses symptom-based clinical algorithms. Nurses assign the call to the most appropriate algorithm and the patient's symptoms determine the questions asked and the action to be taken following the call, which could be guidance on self-care or referral to their general practitioner (GP) or advice to attend a hospital emergency department. Anonymised data on the number of calls for key algorithms are sent to the Health Protection Agency (HPA) Real-time Syndromic Surveillance Team every day for surveillance purposes. As the number of daily calls to NHS Direct varies, indicators are expressed as the percentage of calls for that algorithm using all NHS Direct calls as the denominator. The algorithms for cold/flu, cough, fever, and difficulty breathing were monitored during the 2009 influenza pandemic on a daily basis. Due to the increasing number of calls received by NHS Direct an additional 'swine flu' algorithm was introduced, which was included in the cold/flu calls in order to capture all pandemic related calls.

Call data for cold/flu were mapped by postcode district in the West Midlands region, following an outbreak of pandemic influenza $\mathrm{A}\left(\mathrm{H}_{1} \mathrm{~N}_{1}\right)_{2} 2009$ in a primary school [9], and also in London following an increase in the number of cases in early June.

\section{HPA/QSurveillance system}

The HPA/QSurveillance system was set up by the University of Nottingham and Egton Medical Information Systems (EMIS; a supplier of general practice computer systems) in collaboration with the HPA $[10,11]$. Over 3,400 general practices with over 23 million patients submit data to the QSurveillance database, covering about $38 \%$ of the UK population. Aggregated data on GP consultations for a range of indicators are automatically uploaded daily from GP practice systems to a central database. Consultation data are based on clinical diagnoses that are recorded as codes on the practice system. Indicators, for example ILI, are defined as collections of clinical diagnosis codes. The surveillance system usually produces weekly reports, but daily reports were also provided throughout the pandemic period. Data are available at national, regional and PCT level.

Daily data for ILI, pneumonia, upper respiratory tract infection (URTI), lower respiratory tract infection (LRTI), ILI with antiviral drugs prescribed, and pneumonia with antibiotics prescribed were monitored during the pandemic. Daily ILI data were mapped by PCT, initially only for the West Midlands and London regions, and later also for other regions when the local ILI rates increased. Weekly mapping at PCT level was later extended to all PCTs in England and continued through the second pandemic wave during the winter of 2009/10.

\section{FIGURE 1}

NHS Direct cold/flu calls for West Midlands and London, summer 2009

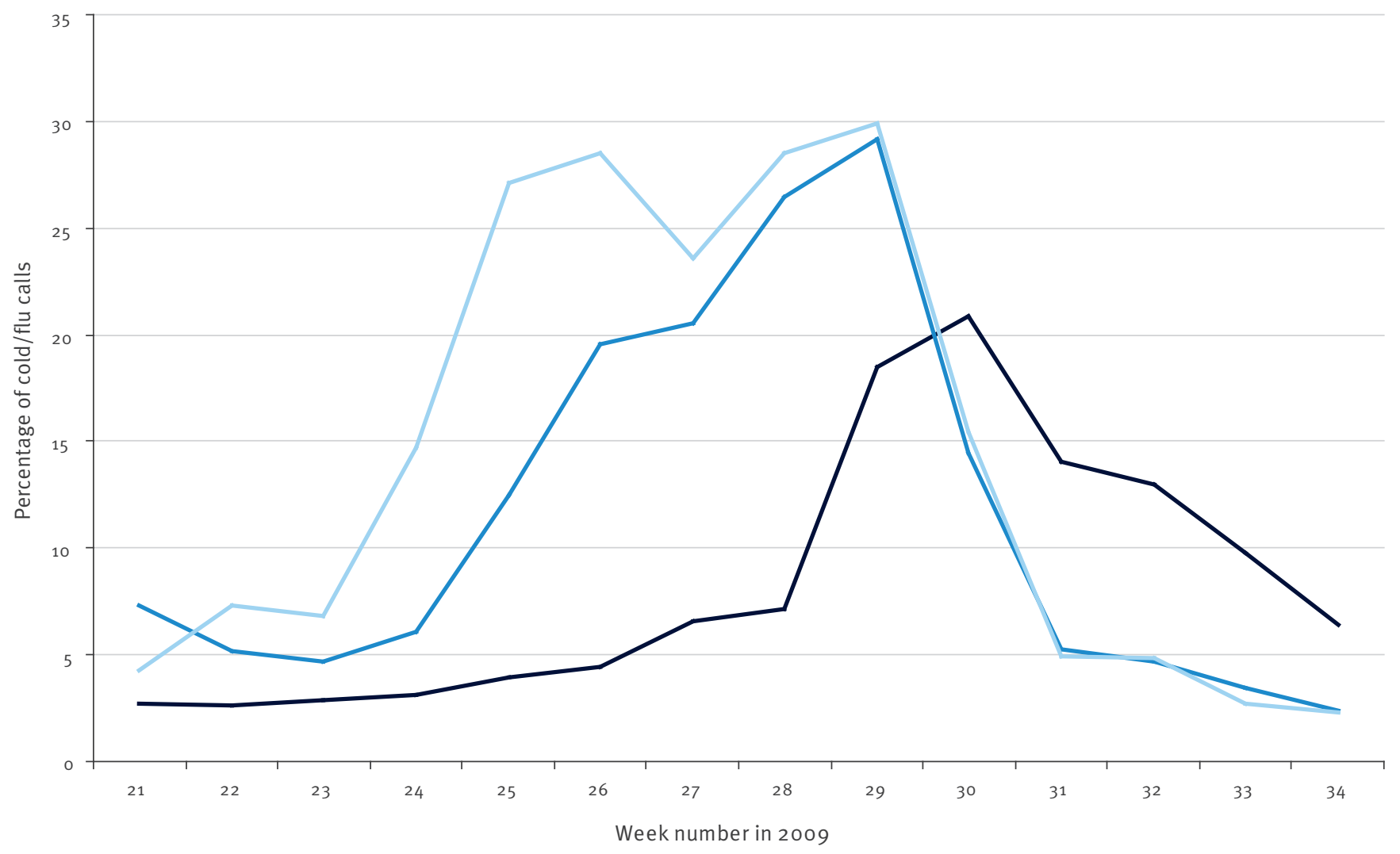

- National London - West Midlands 
The ILI indicator is a group of clinical diagnosis codes recorded by GPs during routine consultations and is widely used as a proxy for community-based influenza activity $[12,13]$. In order to compare ILI rates with the seasonal influenza activity experienced in a normal winter season estimated thresholds for daily and weekly HPA/QSurveillance data were developed and used to interpret ILI data included in surveillance bulletins and PCT maps [11]. All maps were drawn using

\section{FIGURE 2}

HPA/QSurveillance general prctitioner consultation rate for influenza-like illness in Primary Care Trusts in the West Midlands (A) and London (B), summer 2009

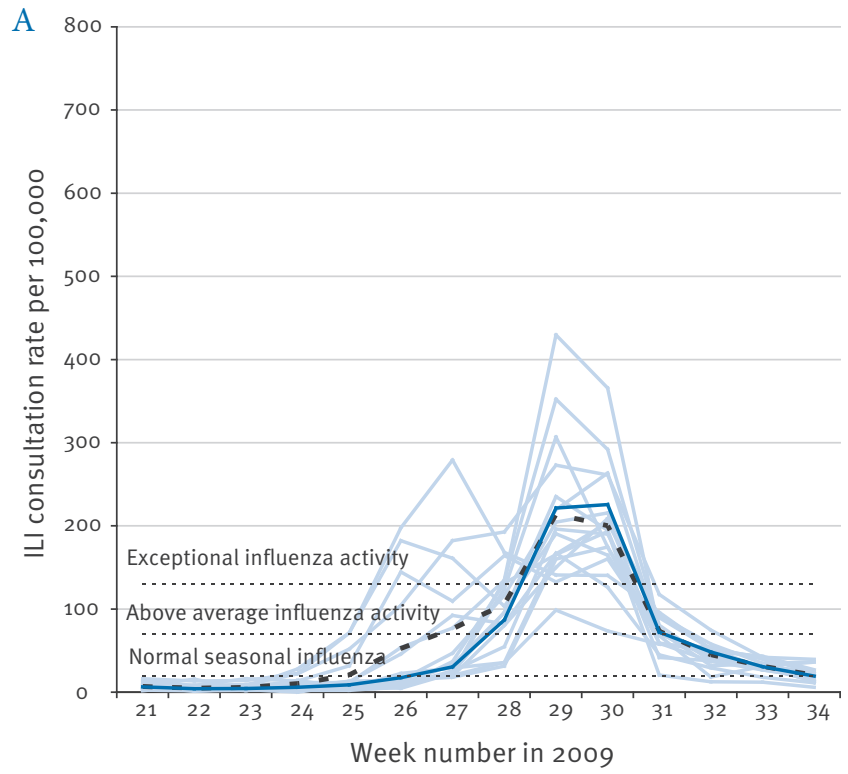

- Primary Care Trusts - - West Midlands —United Kingdom

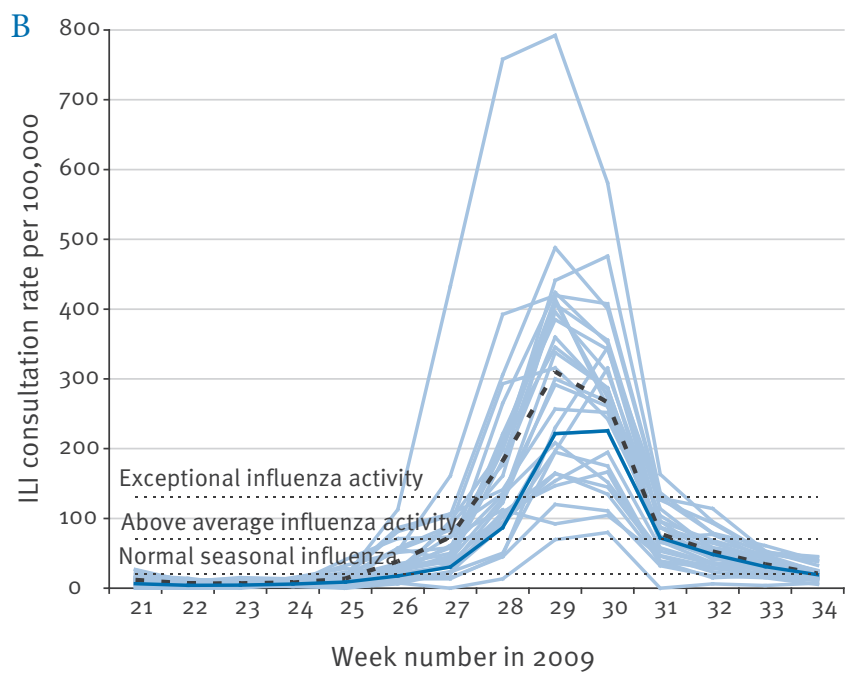

- Primary Care Trusts - - London _ United Kingdom

HPA: Health Protection Agency; ILI: influenza-like illness.

..... Indicative estimated thresholds for QSurveillance weekly influenza-like illness data in the United Kingdom

HPA/QSurveillance system influenza-like illness thresholds

[11]: baseline influenza activity: below 20 per 100,000; normal

influenza activity: 20-70 per 100,000; above average influenza

activity: 70-130 per 100,000; exceptional influenza activity: $\geq 130$ per 100,000
MapInfo Professional version 9.5. In this paper data are presented from week 21 in 2009 (week commencing 18 May), when the first school outbreak occurred in Birmingham, to week 34 in 2009 (week commencing 17 August), when UK ILI rates returned to baseline activity, to demonstrate the progression of the first wave of the influenza pandemic in the UK. This period coincides with the treatment only phase of the outbreak that began on 2 July (in week 27, the week commencing 29 June).

The HPA routinely analyse and monitor syndromic data throughout the year. From the start of the pandemic the HPA Real-time Syndromic Surveillance Team used daily outputs from the HPA/NHS Direct and HPA/ QSurveillance systems to monitor a range of indicators that might suggest wider community transmission of pandemic influenza $A\left(\mathrm{H}_{1} \mathrm{~N}_{1}\right)_{2} 2009$, and were also used, along with laboratory data and local intelligence, to help identify hotspots, areas of particularly high influenza activity and of rapid increase in influenza rates. Data at national, regional (Strategic Health Authority), local health district (PCT), and postcode district level were included in daily bulletins distributed to the HPA, the Department of Health, the National Health Service (NHS) and the Government.

\section{Results}

The first suggestion of community spread was seen in the West Midlands region following an outbreak in a primary school in the Heart of Birmingham PCT where the first case of pandemic influenza $\mathrm{A}\left(\mathrm{H}_{1} \mathrm{~N}_{1}\right)_{2009}$ was confirmed during week 21, 2009 [9]. The cold/flu call data from the HPA/NHS Direct system and the PCT level data from the HPA/QSurveillance system showed two distinct peaks of pandemic influenza activity in the West Midlands (Figures 1 and 2). NHS Direct cold/ flu calls for the West Midlands showed an early rise in calls that peaked in week 26 (week commencing 22 June). There was a second peak in both systems in week 29 (week commencing 13 July). These peaks were respectively four weeks and one week ahead of the national peak in week 30 (week commencing 20 July). In the HPA/QSurveillance system, GP consultation rates for ILI showed that the early increase was accounted for by four PCTs: Heart of Birmingham, where the initial school outbreak occurred, and the three surrounding PCTs, Birmingham East and North, Sandwell, and South Birmingham. By week 26, all four had reached exceptional levels of influenza activity (above 130 consultations per 100,000) except South Birmingham which reached this level in week 27.

Community transmission in London started slightly later and showed a different pattern, with HPA/NHS Direct and HPA/QSurveillance systems both showing a single peak in week 29, the same week as the West Midlands peak, one week ahead of the national peak (Figures 1 and 2). HPA/QSurveillance ILI rates reached exceptional levels in the Tower Hamlets PCT and the City and Hackney PCT in week 27, and the majority of 

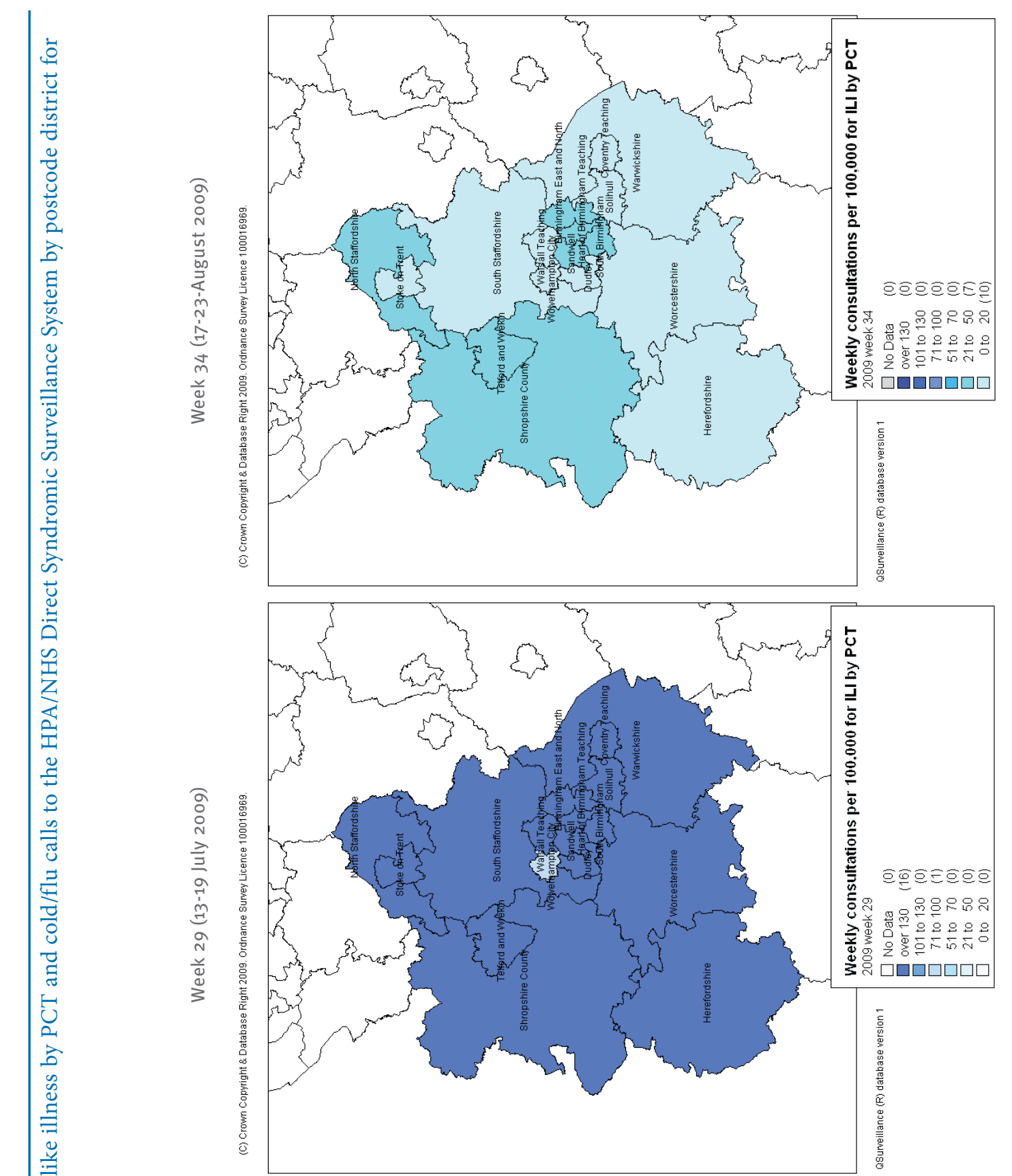

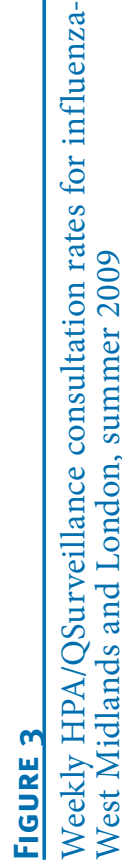

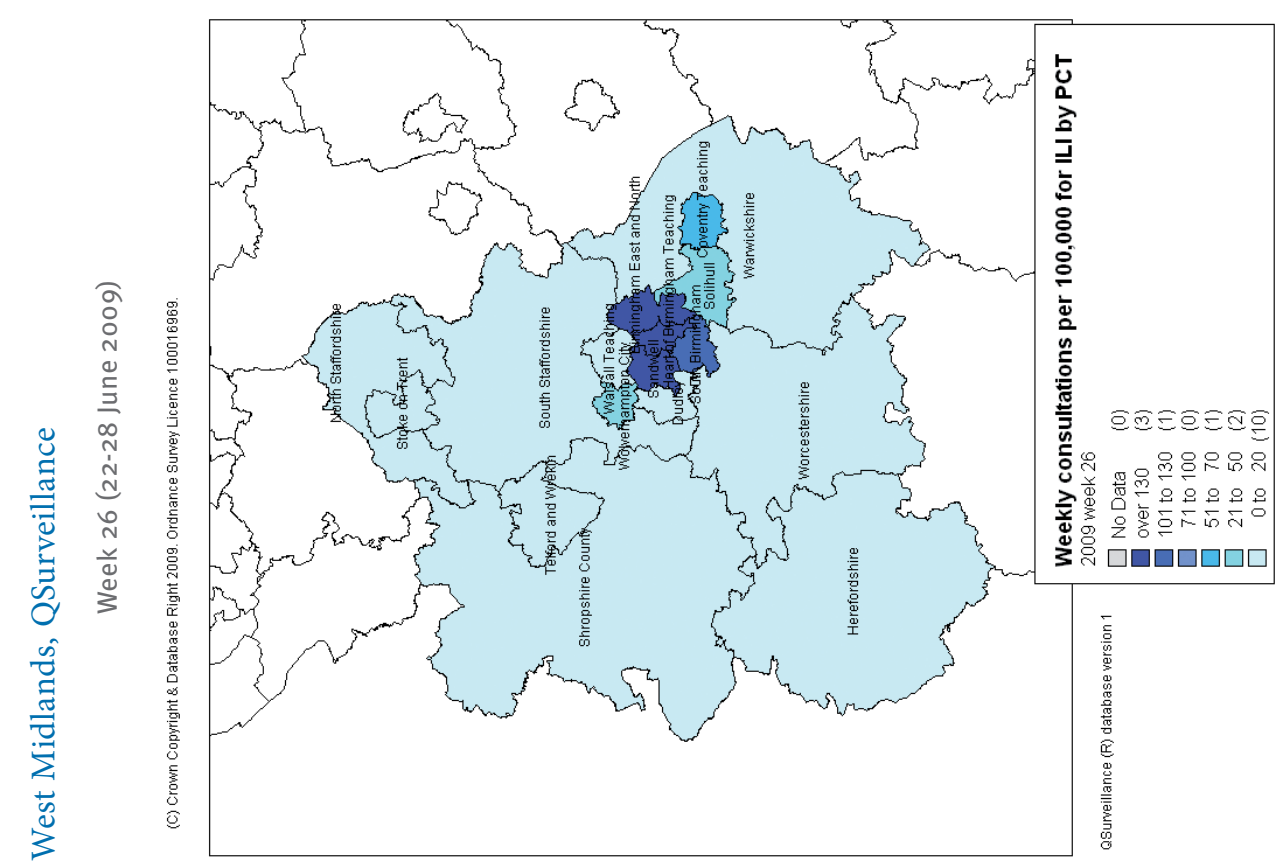



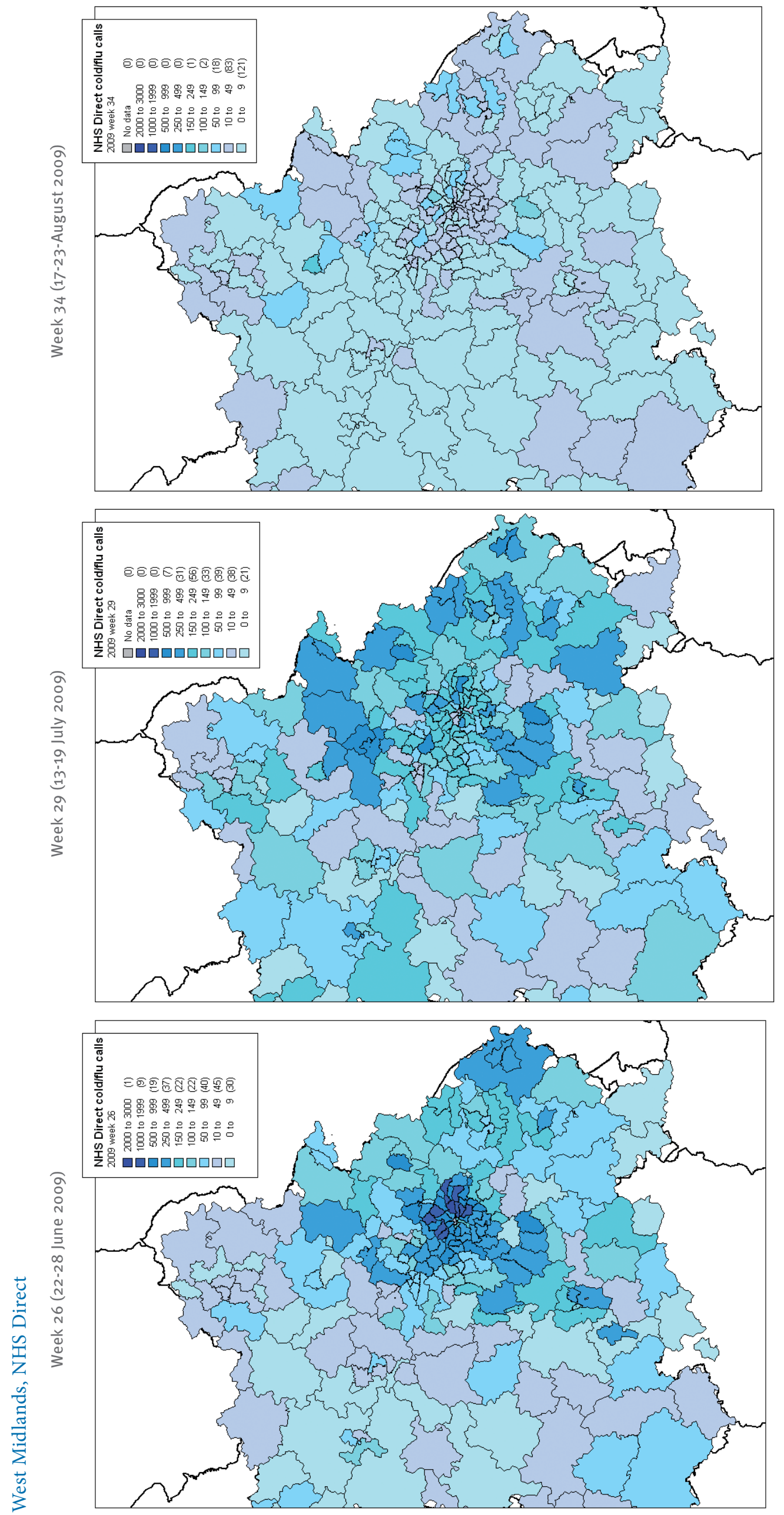


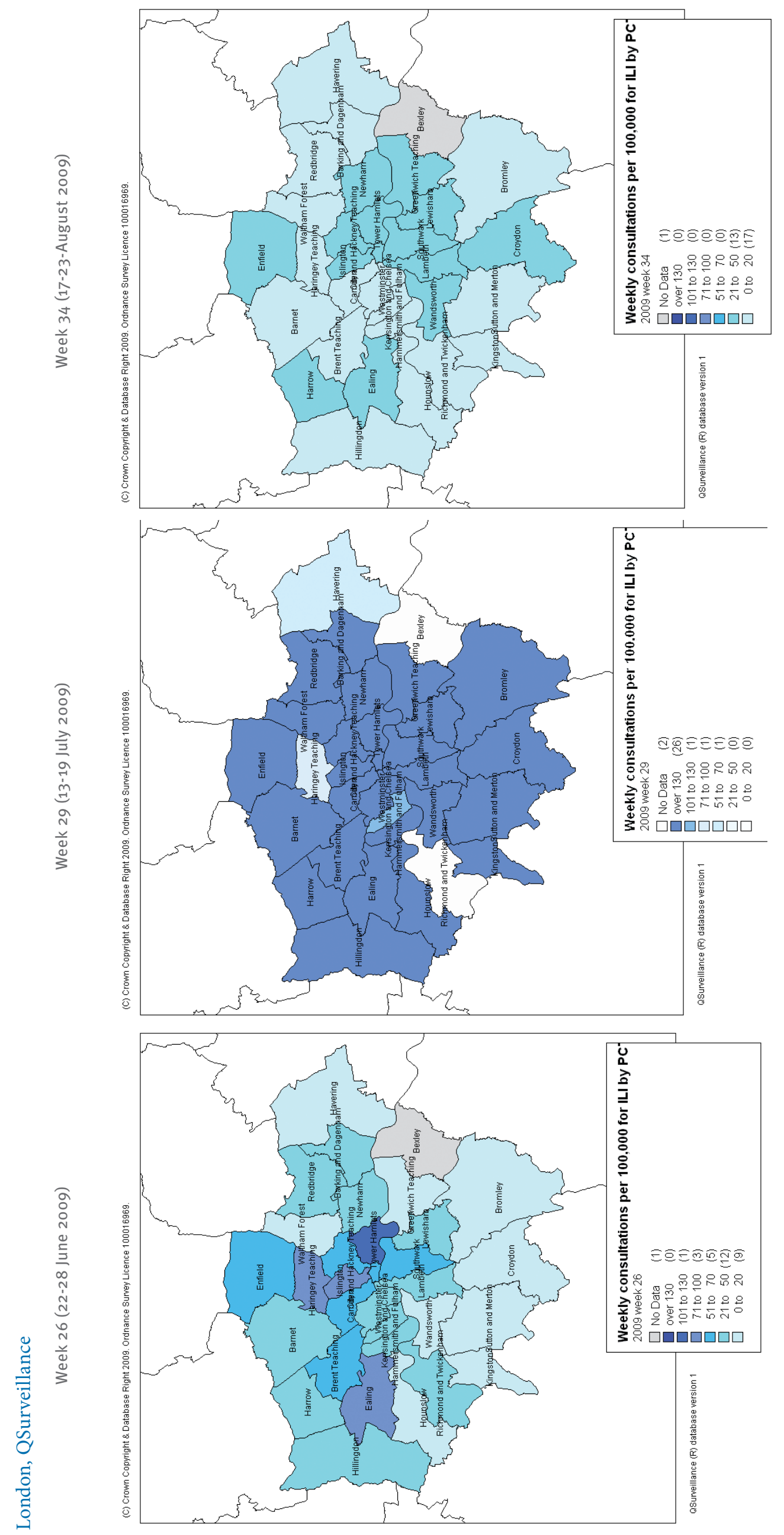




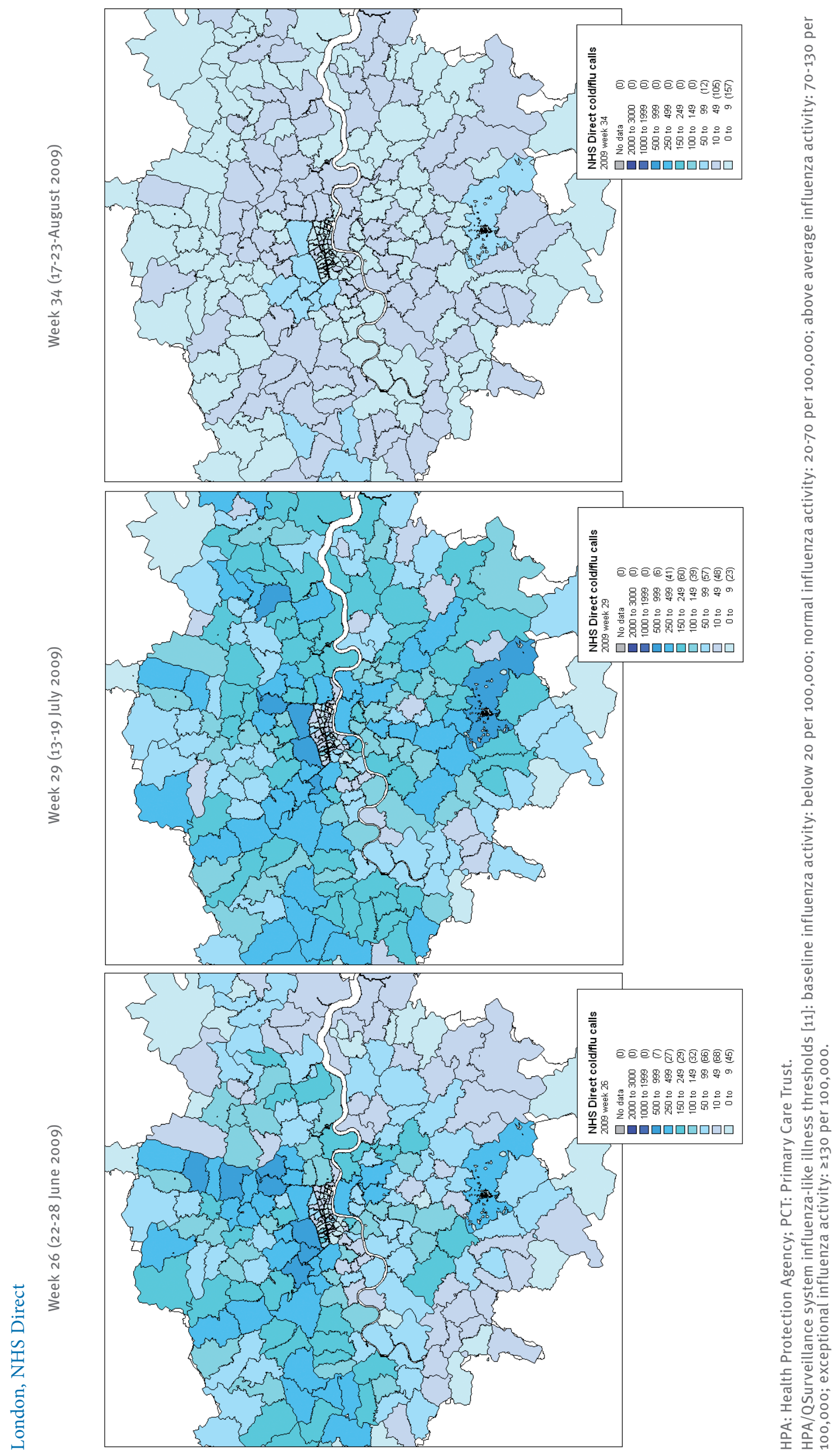


London PCTs simultaneously peaked in week 29. The peak ILI rates in London were generally higher than those seen in the West Midlands, with the highest ILI rates recorded in the Tower Hamlets PCT (792.4 per 100,000 in week 29).

HPA/NHS Direct cold/flu calls were mapped by postcode and HPA/QSurveillance ILI data were mapped by PCT to monitor the geographical spread of the outbreak, in order to assist in the identification of hotspot areas and in the outbreak management, and in directing public health resources (Figure 3). On 19 June 2009 sustained community transmission was declared in the PCTs Birmingham East and North, Heart of Birmingham, South Birmingham, and Sandwell due to high numbers of confirmed cases that were predominantly not travelrelated [11], school absenteeism, high GP consultation rates (HPA/QSurveillance system) and high numbers of calls to NHS Direct.

\section{Discussion}

We used syndromic surveillance systems to track the progress of pandemic influenza $A\left(\mathrm{H}_{1} \mathrm{~N}_{1}\right)_{2009}$ in the UK on a daily basis and were able to show the early stages of community transmission at a local level in the West Midlands and London. These systems were key in defining the start of community transmission. The first evidence of sustained community transmission was seen in the West Midlands. Influenza activity in the West Midlands and London peaked a week ahead of the rest of the UK. Although this hasn't been formally analysed, we can say empirically that there was considerable agreement between data from the HPA/ NHS Direct and HPA/QSurveillance systems, however NHS Direct call data showed an increase a week earlier than the GP consultation data in the HPA/QSurveillance system, confirming the usefulness of NHS Direct as an early warning of outbreaks [6].

HPA/NHS Direct call data were mapped at postcode level and HPA/QSurveillance data were mapped at PCT level. Such maps were used by those managing the incident at national, regional and local levels. Syndromic surveillance data from both systems, along with laboratory data and local intelligence, helped identify hotspots in the early stages of community transmission, and monitor the progress of the outbreak at local level. The data were included in surveillance bulletins and thus influenced the local management of the pandemic.

\section{Limitations of the data}

Although the HPA/QSurveillance system has good coverage in England, there are variations in coverage at local level. The QSurveillance database only collects data from GP practices that use the EMIS practice information system; the coverage at PCT level can therefore vary depending on the number of practices that use that system. Data at PCT level are suppressed if fewer than three practices report to the system in order to preserve the anonymity of patients and practices; data were unavailable for one PCT in London for this reason.

It has been shown that older people and ethnic minorities are less likely to use NHS Direct [14]. While this does not substantially affect the usefulness of regional and national data, this would be important at postcode level and could potentially be a cause of under-reporting for example in a district with a high ethnic minority population. In the context of our study, age was considered a less important limitation because pandemic influenza $A\left(\mathrm{H}_{1} \mathrm{~N}_{1}\right) 2009$ predominately affected younger age groups [15].

The peak of the first wave of the pandemic in the UK in week 30 coincided with the launch of the National Pandemic Flu Service on 23 July 2009, which was established to authorise antiviral drugs for patients who met the clinical criteria for pandemic influenza $\mathrm{A}\left(\mathrm{H}_{1} \mathrm{~N}_{1}\right) 2009$ and thereby remove the pressure from GP practices and NHS Direct. It is likely that this explains at least partly the observed reduction in GP consultation rates for ILI and NHS Direct cold/flu calls in week 31 in 2009 [11]. The highest rates of pandemic influenza $A\left(\mathrm{H}_{1} \mathrm{~N}_{1}\right) 2009$ were seen in school-aged children. During week 30 in 2009 schools closed for the summer holidays, which would have interrupted transmission in that age group and contributed to decreased consultation rates in week 31 of $2009[16,17]$.

\section{Conclusion}

This work has demonstrated the usefulness of local mapping of syndromic surveillance data for the detection of increasing transmission and for the epidemiological description of the pandemic. We detected early rises of pandemic influenza $A\left(\mathrm{H}_{1} \mathrm{~N}_{1}\right)_{2009}$ in the West Midlands and London using these systems. It has prompted further spatio-temporal work to describe in more detail the determinants of the initial spread.

\section{Acknowledgements}

We thank NHS Direct for the call data and the University of Nottingham, EMIS and the EMIS practices for the QSurveillance data extraction.

\section{References}

1. Health Protection Agency and Health Protection Scotland new influenza $A\left(\mathrm{H}_{1} \mathrm{~N}_{1}\right)$ investigation teams. Epidemiology of new influenza $A\left(\mathrm{H}_{1} \mathrm{~N}_{1}\right)$ in the United Kingdom, April - May 2009. Euro Surveill. 2009;14(19):pii=19213. Available from: http:// www.eurosurveillance.org/ViewArticle.aspx?Articleld $=19213$

2. Nicoll A, Coulombier D. Europe's initial experience with pandemic ( $\left.\mathrm{H}_{1} \mathrm{~N}_{1}\right) 2009$ - mitigation and delaying policies and practices . Euro Surveill. 2009;14(29): pii=19279. Available from: http://www.eurosurveillance.org/ViewArticle. aspx?Articleld $=19279$

3. Henning KJ. What is syndromic surveillance? MMWR Morb Mortal Wkly Rep. 2004;53 Suppl:5-11.

4. Fleming DM. Weekly Returns Service of the Royal College of General Practitioners. Commun Dis Public Health. 1999;2(2):96-100. 
5. Elliot AJ, Fleming DM. Surveillance of influenza-like illness in England and Wales during 1966-2006. Euro Surveill. 2006;11(10):pii=651. Available from: http://www. eurosurveillance.org/ViewArticle.aspx?Articleld=651

6. Cooper DL, Verlander NQ, Elliot AJ, Joseph CA, Smith GE. Can syndromic thresholds provide early warning of national influenza outbreaks? J Public Health (Oxf). 2009;31(1):17-25

7. Loveridge P, Cooper D, Elliot AJ, Harris J, Gray J, Large S, et al. Vomiting calls to NHS Direct provide an early warning of norovirus outbreaks in hospitals. J Hosp Infect. 74(4):385-93.

8. Cooper DL, Smith GE, Hollyoak VA, Joseph CA, Johnson $\mathrm{L}$, Chaloner R. Use of NHS Direct calls for surveillance of influenza--a second year's experience. Comm Dis Public Health. 2002;5(2):127-31.

9. Health Protection Agency West Midlands $\mathrm{H}_{1} \mathrm{~N}_{1}$ v Investigation Team. Preliminary descriptive epidemiology of a large school outbreak of influenza $\mathrm{A}\left(\mathrm{H}_{1} \mathrm{~N}_{1}\right) \mathrm{v}$ in the West Midlands, United Kingdom, May 2009. Euro Surveill. 2009;14(27): pii=19264. Available from: http://www.eurosurveillance.org/ViewArticle. aspx?Articleld $=19264$

10. QSurveillance [Internet]. 2010. Available from: www. qsurveillance.org

11. Health Protection Agency (HPA). Epidemiological report of pandemic $\left(\mathrm{H}_{1} \mathrm{~N}_{1}\right) 2009$ in the UK. London: HPA; October 2010. Available from: http://www.hpa.org.uk/web/ HPAweb\&HPAwebStandard/HPAweb_C/1284475323858

12. Zambon MC, Stockton JD, Clewley JP, Fleming DM. Contribution of influenza and respiratory syncytial virus to community cases of influenza-like illness: an observational study. Lancet 2001;358(9291):1410-6.

13. Fleming DM, Elliot AJ, Cross KW. Morbidity profiles of patients consulting during influenza and respiratory syncytial virus active periods. Epidemiol Infect. 2007;135(7):1099-108.

14. Cooper DL, Verlander NQ, Smith GE, Charlett A, Gerard E, Willocks L, et al. Can syndromic surveillance data detect local outbreaks of communicable disease? A model using a historical cryptosporidiosis outbreak. Epidemiol Infect. 2006;134(1):13-20.

15. Miller E, Hoschler K, Hardelid P, Stanford E, Andrews N, Zambon M. Incidence of 2009 pandemic influenza A H1N1 infection in England: a cross-sectional serological study. Lancet. 2010;375(9720):1100-8.

16. Ferguson NM, Cummings DA, Fraser C, Cajka JC, Cooley PC, Burke DS. Strategies for mitigating an influenza pandemic. Nature. 2006;442(7101):448-52.

17. Ghani AC, Baguelin M, Griffin J, Flasche S, Pebody R, van Hoek AJ, et al. The early transmission dynamics of $\mathrm{H}_{1} \mathrm{~N}_{1} \mathrm{pdm}$ influenza in the United Kingdom. PLoS Curr. 2009; RRN1130. doi:10.1371/currents.RRN1130. 